\title{
Correlation between pre-systolic forward flow and diastolic function
}

\begin{abstract}
Background: Left ventricular out flow tract (LVOT) pre-systolic forward flow by spectral Doppler is an interesting phenomenon (figure1). However, its significance is not well defined. Our study aims to examine echo characteristics of subjects with positive PSW and correlation between PSW velocity and diastolic parameters.
\end{abstract}

Methods: We conducted a prospective study recruiting 91 subjects with positive presystolic forward flow by pulsed wave Doppler. Correlation coefficient utilizing Pearson method was conducted between pre-systolic wave velocity and left atrial (LA) volume index, E wave deceleration time, average e'velocity (septal and lateral).

Result: No significant correlation was found.

Conclusion: Pre-systolic wave is found in many normal subjects and is explained by the left ventricular fluid mechanics of ring of vortex model.

Keywords: pre-systolic wave, diastolic function, diastolic flow, echocardiography, left ventricular, ejection fraction, adverse, cardiac
Volume 5 Issue 4 - 2016

\author{
Sami Nimer Ghazal,' Jawaher Towhari² \\ 'Internal Medicine Department, College of Medicine, University \\ of Dammam, Saudi Arabia \\ ${ }^{2}$ College of Cardiac Technology, University of Dammam, Saudi \\ Arabia
}

Correspondence: Sami Nimer Ghazal, Cardiology division, Internal Medicine department, King Fahd Hospital of the University, University of Dammam, Al-Khobar 31952, Saudi Arabia,Emailsghazal@uod.edu.sa

Received: March 20, 2016 | Published: April 20, 2016
Abbreviations: LVOT, left ventricular outflow tract; PSW, pre-systolic wave; PW, pulsed wave; MACE, major adverse cardiac events; LVEDP, left ventricular end diastolic pressure; EF, ejection fraction

\section{Introduction}

Left ventricular out flow tract (LVOT) pre-systolic forward flow by spectral Doppler is an interesting phenomenon (Figure 1). However, its significance is not well defined. It was classically suggested to be caused by stiff ventricle and elevated left ventricular end diastolic pressure (LVEDP) as atrial contraction will further increase the pressure in the left ventricle causing early premature forward flow across the aortic valve. However, that was not proved.
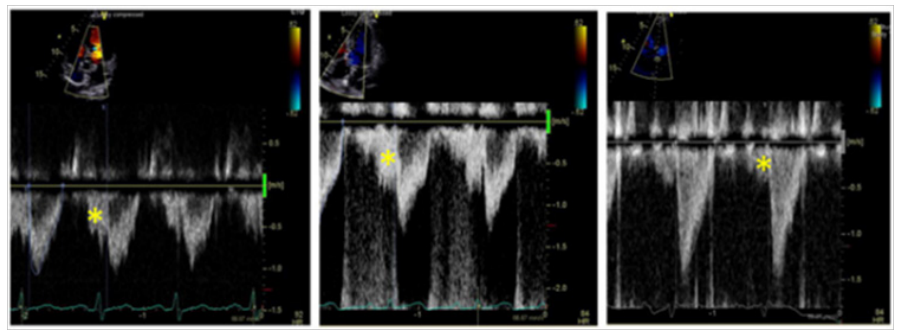

Figure I Pulsed wave spectral Doppler showing pre-systolic forward flow (asterisks).

In 1990 Panaviotou \& Byrd $^{1}$ studied this phenomenon in 59 patients and concluded that pre-systolic forward flow is a normal phenomenon found in the majority of normal subjects and suggested that the fluid mechanics of left ventricular outflow as a ring vortex is the responsible mechanism. ${ }^{1}$ Moreover, it was found in another more recent study that absence of pre-systolic wave (PSW) was associated with reduced left ventricular ejection fraction $(\mathrm{EF})$ and increased major adverse cardiac events (MACE). ${ }^{2}$ Our study aims to examine echo characteristics of subjects with PSWand the correlation between PSW velocity and parameters of diastolic function and LVEDP, namely left atrial (LA) volume index, E wave deceleration time, average e' velocity(septal and lateral) and E/e' ratio.

\section{Material and methods}

We prospectively studied 91consecutivepatients undergoing routine transthoracic echocardiography acquired at our institute starting on May 11, 2015 till July 7, 2015. Only patients with technically good quality studies were included. Inclusion criterion is the presence of LVOT diastolic forward flow by pulsed wave (PW) Doppler in apical 5 or 3 chamber views.

Exclusion criteria were

i. Presence of moderate or more mitral stenosis.

ii. Presence of moderate or more mitral regurgitation;

iii. Significant mitral annular calcification;

iv. Patient with incomplete echo cardio graphic data.

Blood pressure was recorded for all patients just prior to the transthoracic echocardiographic examination. Left atrial volume was measured utilizing biplane method of disks and indexed to the body surface area. Doppler echocardiographic measurements were performed according to The European Association of Echocardiography/American Society of Echocardiography recommendations for the evaluation of diastolic function. ${ }^{3}$ Mitral annular velocities were obtained from PW tissue Doppler imaging performed in the apical 4 chamber views. Septal e'velocity and lateral e'velocities were measured. Accordingly, septal, lateral and average E/e' ratios were calculated. PW Doppler of the LVOT was obtained by placing the PW sample volume in the LVOT, approximately $1 \mathrm{~cm}$ from the aortic valve. Peak velocity of PSW was measured. Mitral E wave peak velocity and deceleration time were obtained from pulse wave Doppler in the apical 4-chamber view at the tip of mitral valve leaflets. Correlation test was performed utilizing Pearson correlation coefficient between PSW velocity and left atrial volume index, E wave deceleration time, average e'velocity and average E/e' ratio.

\section{Results}

91 patients with PSW by PW Doppler were recruited. Baseline 
characteristics (Table 1) showed mean age of 56.9 years. All patients were in normal sinus rhythm with mean heart rate of $74 \mathrm{bpm}$. Mean systolic and diastolic blood pressure were $137 \mathrm{mmHg}$ and $78 \mathrm{mmHg}$ respectively. Mean indexed left atrial volume was $33 \mathrm{ml} / \mathrm{m}^{2}$. Mean velocity of the PSW is $43 \mathrm{~cm} / \mathrm{s}$ and $14(15 \%)$ patients of them had high E/e' ratio defined as average E/e' more than 13. Correlation coefficients is represented as (r) in table 2.

Table I Baseline Characteristics of patient with positive presystolic wave by PW

\begin{tabular}{|c|c|}
\hline Variable & $\mathbf{n}=91$ \\
\hline Mean age (years) $\pm S D$ & $56 \pm 15$ \\
\hline Mean systolic Blood Pressure \pm SD & $|38 \pm 2|$ \\
\hline Mean diastolic Blood Pressure \pm SD & $80 \pm 11$ \\
\hline Mean heart Rate $(\mathrm{bpm}) \pm \mathrm{SD}$ & $75 \pm 14$ \\
\hline Mean LA volume index $(\mathrm{ml} / \mathrm{m} 2) \pm S D$ & $32 \pm 13$ \\
\hline Mean $\mathrm{E}$ wave deceleration time ( $\mathrm{m} \mathrm{sec}$ ) & $206 \pm 64$ \\
\hline Mean e' septal velocity $(\mathrm{cm} / \mathrm{sec}) \pm \mathrm{SD}$ & $8 \pm 3$ \\
\hline Mean e' lateral velocity $(\mathrm{cm} / \mathrm{sec}) \pm S D$ & $9 \pm 4$ \\
\hline
\end{tabular}

Table 2 Correlation of pre-systolic forward flow velocity by pulsed wave Doppler

\begin{tabular}{lll}
\hline Variable & Correlation Coefficient $(\boldsymbol{r})$ & P-Value \\
\hline Average E/e' & 0.126 & 0.239 \\
E wave deceleration time & 0.237 & 0.027 \\
Average e' & 0.045 & 0.671 \\
LA volume index & 0.121 & 0.259 \\
\hline
\end{tabular}

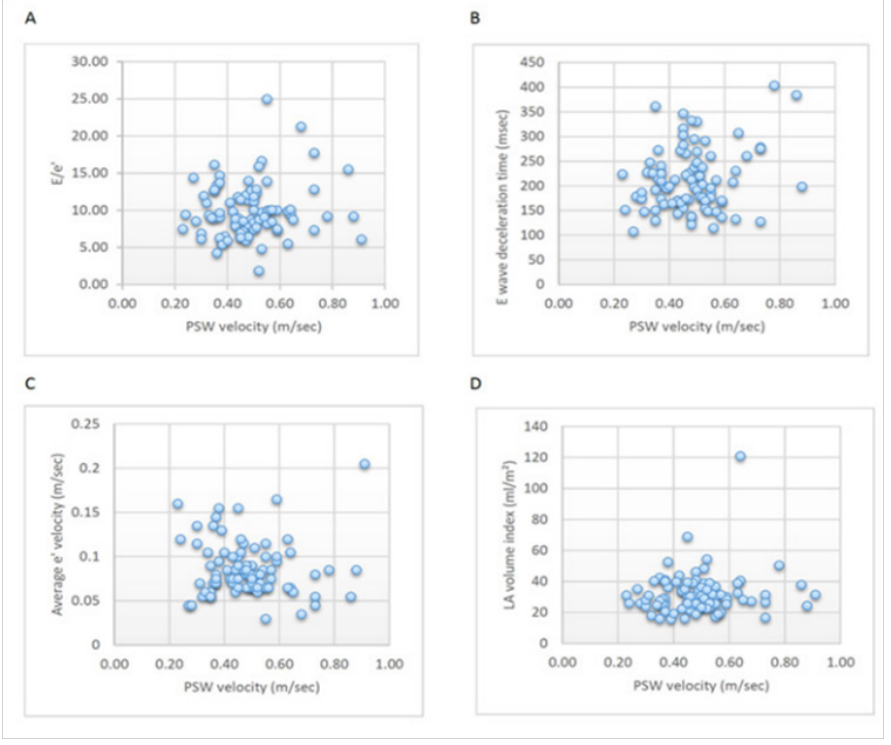

Figure 2 Scatterplot showing no significant correlation between pre-systolic forward flow velocity and average E/e' (A), E wave deceleration time (B), average mitral annular velocity (C) and LA volume index (D).

\section{Discussion}

E/e' ratio is a validated and reproducible method of estimation of LV filling pressure. E/e' $>15$ associated with left ventricular end diastolic pressure (LVEDP)> 20mmHg. ${ }^{4}$ LA volume is another sensitive marker of elevated LVEDP. ${ }^{5} \mathrm{E}$ wave deceleration time and mitral annular velocities are among the most important recommended parameters of to evaluate for diastolic function estimation ${ }^{3} \mathrm{We}$ studied the correlation between PSW magnitude (velocity) and fore mentioned variables of diastolic function and LVEDP and we did not find any significant correlation (Figure 2). Therefore, it became clear that presence of PSW is not related to the compliance and diastolic function of the left ventriclenor to LVEDP. PSW is more or less a normal phenomenon.

The fluid mechanics of LV filling were extensively studied and found to simulate a ring vortex model (Figure 3). ${ }^{6}$ Our findings of no significant correlation between PSW and diastolic parameters does not contradict the explanation of ring vortex fluid mechanics being the responsible cause forPSW, instead, we further support this explanation. The association between absence of PSW and increased MACE is due todisrupted fluid mechanics in dilated and failing heart.

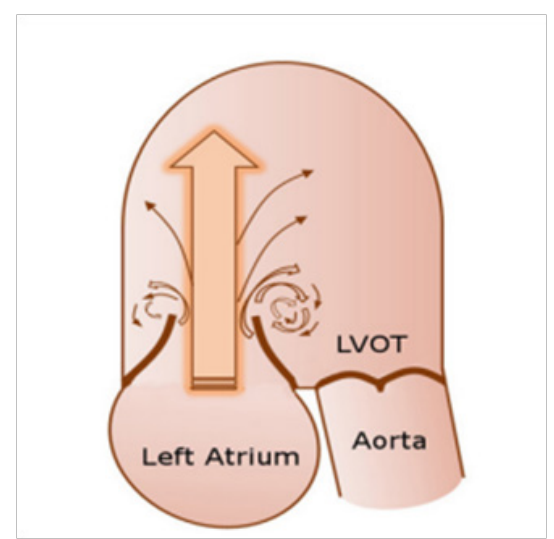

Figure 3 LV fluid mechanics as a ring vortex model.

\section{Conclusion}

PSW is found in many normal subjects and is a normal phenomenon. Fluid mechanics of ring vortex is an acceptable explanation of the mechanism of PSW. No significant correlation was found between magnitude of PSW and diastolic function. Absence of PSW was found to be more prevalent in reduced EF patient and thus was associated with increased MACE.

\section{Acknowledgments}

None.

\section{Conflicts of interest}

Authors declare that there is no conflict of interest.

\section{References}

1. Panaviotou H, Byrd BF. Origin and significance of diastolic Doppler flow signal in the Left ventricular outflow tract. J Am Coll Cardiol. 1990;16(7):1625-1631.

2. Joshi KR, Kabirdas D, Romero CA, et al. Clinical Significance of a Presystolic Wave. Am J Cardiol. 2014;114(10):1599-1602.

3. Nagueh S, Appleton C, Gillebert T, et al. Recommendations for the Evaluation of Left Ventricular Diastolic Function by Echocardiography. J Am Soc Echocardiogr. 2009;22(2):107-133.

4. Ommen SR, Nishimura RA, Appleton CP, et al. Clinical utility of Doppler echocardiography and tissue Doppler imaging in the estimation of left ventricular filling pressures: A comparative simultaneous Doppler-catheterization study. Circulation. 2000;102(15):1788-1794.

5. Tsang TS, Barnes ME, Gersh BJ, et al. Left atrial volume as a morphophysiologic expression of left ventricular diastolic dysfunction and relation to cardiovascular risk burden. Am J Cardiol. 2002;90(12):1284-1289.

6. Bellhouse BJ. Fluid mechanics of a model mitral valve and left ventricle. Cardiovasc Res. 1972;6(2):199-210. 